\title{
Efeito inibitório da via eferente auditiva no transtorno de processamento auditivo central
}

\section{Auditory efferent inhibitory effect in the central auditory processing}

\section{disorder}

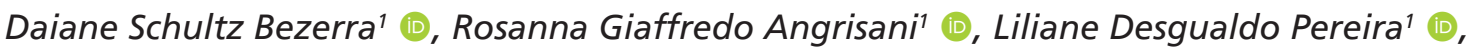 \\ Marisa Frasson de Azevedo ${ }^{1}$ (D), Karin Ziliotto Dias ${ }^{1}$
}

\section{RESUMO}

Objetivo: verificar e comparar a ocorrência e magnitude do efeito inibitório da via auditiva eferente em crianças e adolescentes com processamento auditivo normal e alterado, identificando um valor de corte para uso na prática clínica. Métodos: estudo prospectivo, com amostra composta por 30 crianças de 7 a 14 anos, sendo 15 com avaliação de processamento auditivo normal (grupo controle) e 15 com processamento auditivo alterado (grupo estudo). Ambos os grupos foram submetidos à anamnese e avaliados por meio dos testes de emissões otoacústicas evocadas por estímulos transientes na ausência e presença de ruído contralateral, avaliação audiológica básica e de processamento auditivo. Resultados: houve menor ocorrência do efeito inibitório da via eferente no grupo estudo ( $\mathrm{p}$-valor $=0,038$ ). Os valores médios obtidos no grupo controle foram 0,71 na orelha direita e 0,87 na orelha esquerda e no grupo estudo, 0,55 na orelha direita e 0,41 na orelha esquerda. Os grupos controle e estudo diferiram de modo significativo na orelha esquerda. Conclusão: houve redução do efeito inibitório da via eferente em crianças e adolescentes com transtorno de processamento auditivo, sugerindo alteração funcional do sistema eferente olivococlear medial. O valor que separou as crianças com e sem alteração do sistema eferente foi de $0,55 \mathrm{~dB}$ na prática clínica, com $70 \%$ de sensibilidade e $66,7 \%$ de especificidade.

Palavras-chave: Audição; Vias auditivas; Emissões otoacústicas espontâneas; Transtorno da percepção auditiva; Vias eferentes

\begin{abstract}
Purpose: Verify and compare the occurrence and magnitude of the inhibitory effect of the efferent auditory pathway in children and adolescents with normal and auditory processing disorder, identifying a cutoff value to use in clinical settings. Methods: A prospective study, with a total sample of 30 children aged 7 to 14 years, half with normal auditory processing assessment (Control Group) and the other half with auditory processing disorder (Study Group). Both groups were submitted to anamnesis and evaluated using the otoacoustic emissions tests evoked by transient stimuli in the absence and presence of contralateral noise, basic audiological evaluation and auditory processing. Results: The study group presented a lower occurrence of the inhibitory effect of the efferent pathway when compared to the control group ( $\mathrm{p}$ value $=0.038$ ). The mean values obtained for the control group were 0.71 in the right ear and 0.87 in the left ear and for the study group, 0.55 in the right ear and 0.41 in the left ear. The two groups showed a statistically significant difference for the left ear. Conclusion: There was a reduction in the inhibitory effect of the efferent pathway in children and adolescents with auditory processing disorder, suggesting a functional change in the medial olivocochlear efferent system. In this study, the cutoff value of $0.55 \mathrm{~dB}$ separated children with and without alteration of the efferent system with $70 \%$ sensitivity and $66.7 \%$ specificity.
\end{abstract}

Keywords: Hearing; Auditory pathways; Spontaneous otoacoustic emissions; Disorder of auditory perception; Efferent pathways

\footnotetext{
Trabalho realizado na Escola Paulista de Medicina, Universidade Federal de São Paulo - UNIFESP - São Paulo (SP), Brasil.

${ }^{1}$ Departamento de Fonoaudiologia, Escola Paulista de Medicina, Universidade Federal de São Paulo - UNIFESP - São Paulo (SP), Brasil.

Conflito de interesses: Não.

Contribuição dos autores: DSB participou da idealização do estudo, coleta, análise e interpretação dos dados e redação do artigo; RGA participou da redação e supervisão do artigo e aprovação da versão final a ser publicada; LDP, MFA e KZD foram responsáveis pela supervisão e aprovação da versão final do artigo a ser publicada; MFA participou, na condição de coorientadora, da idealização do estudo, análise e interpretação dos dados; KZD participou, na condição de orientadora, da idealização do estudo, análise e interpretação dos dados e redação do artigo.

Financiamento: Nada a declarar.
}

Autor correspondente: Rosanna Giaffredo Angrisani. E-mail: roangrisani@gmail.com

Recebido: Novembro 13, 2020; Aceito: Março 24, 2021 


\section{INTRODUÇÃO}

A audição é fundamental para a aquisição da comunicação oral. A integridade anátomo funcional do sistema auditivo periférico e central, somada à exposição a experiências auditivas no ambiente, constitui um pré-requisito para aquisição e desenvolvimento normal da linguagem ${ }^{(1)}$.

O processamento auditivo da informação acústica é altamente complexo e redundante, pois requer muitos mecanismos neurofisiológicos e cognitivos, a fim de que ocorra sua precisa decodificação. $\mathrm{O}$ ato de ouvir refere-se à capacidade de detectar, analisar e organizar tais informações, processos esses que abrangem habilidades para o reconhecimento, discriminação e localização dos sons, além de memorização e integração, envolvendo estímulos acústicos não verbais simples, bem como os mais complexos, como, por exemplo, a fala ${ }^{(2,3)}$.

A investigação do efeito inibitório da via eferente (EIVE), também denominado efeito de supressão das emissões otoacústicas, permite a obtenção de informações sobre o funcionamento do sistema eferente olivococlear medial (SEOCM), cuja função pode ser avaliada de forma indireta, por meio da análise das emissões otoacústicas (EOA) registradas na presença de ruído simultâneo. O sistema eferente é acionado pela presença de ruído de banda larga, que reduz a contração das células ciliadas externas, diminuindo a resposta das $\mathrm{EOA}^{(2,4)}$.

O EIVE tem uma função importante em tarefas de discriminação auditiva, de seletividade de frequências altas e de inteligibilidade das frequências de fala, principalmente em ambientes ruidosos. A literatura aponta que, em geral, indivíduos com dificuldades de aprendizado, distúrbios de linguagem, gagueira e pessoas com dificuldades na discriminação de fala no ruído têm diminuição ou ausência do efeito inibitório da via eferente sobre as emissões otoacústicas ${ }^{(2,4,5)}$.

Uma das principais queixas das crianças com transtorno de processamento auditivo central (TPAC) consiste na dificuldade de inteligibilidade de fala em ambientes ruidosos e estudos indicam o impacto negativo que a dificuldade de percepção auditiva tem na comunicação, no aprendizado, no desempenho escolar e na vida social desses indivíduos ${ }^{(3,6)}$.

$\mathrm{Na}$ literatura, alguns estudos levantaram a hipótese de que muitas dessas crianças poderiam apresentar alteração do efeito inibitório da via eferente. ${ }^{(6-10)}$. O esclarecimento do papel e da associação do EIVE com o TPAC poderia nortear e oferecer opções de reabilitação no tratamento desse transtorno ${ }^{(6-10)}$.

Pressupõe-se que o estudo do efeito inibitório da via eferente em crianças com transtorno do processamento auditivo central poderia contribuir para o maior conhecimento sobre a função eferente da via auditiva e seu papel na escuta em ambientes ruidosos, principalmente durante o período de desenvolvimento infantil.

A investigação do EIVE pode tornar-se mais um instrumento preciso e confiável para auxiliar na identificação e intervenção oportuna nos mecanismos envolvidos no TPAC, impactando positivamente na saúde auditiva e na vida social dessa população. Na literatura, estudo sugere a inclusão da avaliação do efeito inibitório da via eferente na avaliação do processamento auditivo $^{(6)}$. Entretanto, na prática clínica, ainda há necessidade de se estabelecer valores de referência que permitam auxiliar na identificação de alterações do sistema auditivo eferente.

Assim, o objetivo do presente estudo foi verificar e comparar a ocorrência e magnitude do efeito inibitório da via auditiva eferente sobre as emissões otoacústicas evocadas por estímulos transientes (EOAT) em crianças e adolescentes com processamento auditivo normal e alterado, estabelecendo um valor de corte para uso em audiologia clínica.

\section{MÉTODO}

Estudo transversal prospectivo realizado em um serviço público de saúde e aprovado pelo Comitê de Ética em Pesquisa em Seres Humanos da Universidade Federal de São Paulo, conforme parecer $n^{\circ}$ 940.099/16.

Os indivíduos convidados para participar do estudo eram provenientes do Ambulatório de Neuroaudiologia e do Ambulatório de Audiologia Infantil, ambos pertencentes ao Hospital Universitário da instituição promotora da pesquisa.

Seguindo os princípios éticos das pesquisas envolvendo seres humanos, as mães e/ou responsáveis pelas crianças, leram e assinaram o Termo de Consentimento Livre Esclarecido, conforme resolução do Conselho Nacional da Saúde n ${ }^{\circ} 466$ de 12 de dezembro de 2012. As crianças e adolescentes alfabetizados leram, esclareceram suas dúvidas e assinaram o Termo de Assentimento.

A amostra foi composta por crianças e adolescentes atendidos no Sistema Único de Saúde (SUS) e provenientes de escola pública, entre os meses de junho e dezembro de 2016. Todos foram submetidos à audiometria tonal liminar, logoaudiometria, imitanciometria, emissões otoacústicas evocadas por estímulos transientes (EOAT) e avaliação do processamento auditivo central (PAC).

Os critérios de inclusão foram: limiares de audibilidade para as frequências sonoras de $250 \mathrm{~Hz}$ a $8000 \mathrm{~Hz}$, menores ou iguais a 20 dBNA; logoaudiometria convencional, composta pelo índice percentual de reconhecimento de fala (IPRF), com resultados iguais ou superiores a $92 \%$ de acertos e limiar de recepção de fala (LRF) compatível com a média tritonal das frequências sonoras de $500 \mathrm{~Hz}, 1000 \mathrm{~Hz}$ e $2000 \mathrm{~Hz}$. Medidas de imitância acústica: timpanometria e pesquisa dos reflexos acústicos no modo contralateral. Considerou-se como critério de normalidade curva timpanométrica do tipo $\mathrm{A}^{(11)}$ e reflexos acústicos presentes no modo contralateral, com intensidade de 70 a 90 dB nível de sensação. Na sequência, foi realizada a avaliação completa do PAC, de acordo com protocolo seguido pela equipe da instituição ${ }^{(12)}$.

Foram excluídos aqueles que apresentavam perdas auditivas, evidências de doenças neurológicas, genéticas e/ou psiquiátricas, ou que já estavam inseridos em programas de treinamento auditivo e/ou terapia fonoaudiológica.

Dessa forma, a amostra foi constituída por 30 crianças de ambos os sexos, sendo 15 (14 do sexo masculino, um do sexo feminino) com TPAC (grupo estudo - GE) e 15 (oito do sexo masculino, sete do sexo feminino) com desenvolvimento típico e avaliação de processamento auditivo normal (grupo controle - GC). A faixa etária variou de 7 a 14 anos, GE com média de idade de 10,1 anos (+- 1,7) e mediana de 11 anos e GC com média de idade de 9,9 anos $(+-1,7)$ e mediana de 10 anos, a fim de se obter homogeneidade amostral entre os grupos, quanto à idade.

A audiometria tonal liminar foi realizada em cabina acústica com audiômetro Madsen Orbiter 922, fones TDH 39 e coxim MX 41- padrão ANSI-69. As medidas de imitância acústica foram registradas com analisador de orelha média Interacoustics, modelo AT - 235. A avaliação do PAC foi realizada em cabina acústica, utilizando audiômetro Madsen Orbiter 922, com compact discs (CDs) contendo estímulos gravados para a realização dos testes de PAC e um CD player portátil, acoplado ao audiômetro, para a apresentação dos estímulos gravados. A avaliação foi realizada por meio dos seguintes testes auditivos: Teste de Localização Sonora (TLS); Teste de Memória Sequencial 
para Sons Verbais (TMSV); Teste de Memória Sequencial para Sons Não Verbais (TMSnV); Teste de Fala com Ruído Branco (F/R); Teste Dicótico de Dígitos (TDD); Teste de Detecção de Intervalos Aleatórios (RGDT - Random Gap Detection Test) e Teste de Reconhecimento de Padrão de Duração com sons de flauta.

A classificação dos testes de PAC em normal e alterado seguiu os critérios recomendados na literatura ${ }^{(12,13)}$ e o diagnóstico de transtorno de processamento auditivo ocorreu todas as vezes em que que um indivíduo apresentava um ou mais testes considerados alterados.

Os testes eletroacústicos constaram da pesquisa das EOAT e do EIVE realizados com o equipamento do sistema ILO V6, fabricado pela Otodynamics, em cabina acústica. Inicialmente, com o objetivo de verificar a integridade da função coclear, já que a presença de resposta é pré-requisito para se avaliar o sistema eferente, pois para se inibir uma resposta ela deve estar presente, foi realizada a pesquisa das emissões otoacústicas com estímulos transientes (EOAT). Para esta etapa, o estímulo utilizado foi clique não linear e intensidade entre 75 e 85 dBpeNPS. Considerou-se presença de EOAT quando a relação sinal/ruído foi maior ou igual a $3 \mathrm{~dB}$ nas bandas de frequências sonoras de 2000 a $4000 \mathrm{~Hz}$, com reprodutibilidade maior ou igual a $70 \%$ e estabilidade do estímulo da sonda maior ou igual a $70 \%$.

Em seguida, realizou-se um segundo procedimento para a pesquisa do EIVE sobre as EOAT. Para tanto, duas sondas (sonda 1 e sonda 2) foram posicionadas, uma em cada orelha. Os estímulos foram apresentados em blocos alternados de dez segundos de cliques lineares a 65 dBNPS (sonda 1) e dez segundos com ruído de banda larga contralateral a 60 dBNPS (sonda 2), até completar 260 séries de estímulos. O cálculo do EIVE foi obtido pelo resultado da subtração da amplitude de resposta das EOAT, comparando a resposta com e sem ruído, utilizando-se cliques lineares. O efeito inibitório da via eferente
(EIVE) foi considerado presente quando houve redução das amplitudes das EOAT de, pelo menos 0,5 dB nível de pressão sonora (NPS) na presença de ruído contralateral ${ }^{(14)}$.

Para a análise estatística dos dados, foram utilizados o Teste de Mann-Whitney para comparar os grupos em relação aos resultados dos testes de PAC e teste Qui-Quadrado para medir a porcentagem de presença ou ausência de EIVE entre os grupos. A análise Receiver Operating Characteristic (ROC) foi a técnica escolhida por ser um método gráfico simples e robusto, que indica os diferentes pontos de corte do teste, segundo seus níveis de sensibilidade (eixo Y) e especificidade (eixo X). As áreas abaixo da curva representam o poder do instrumento para classificar corretamente os indivíduos sadios e os doentes ${ }^{(15)}$.

Para este trabalho, foi definido um nível de significância (p-valor) de 0,05 (5\%). Os softwares utilizados para esta análise estatística foram: SPSS V20, Minitab 16 e Excel Office 2010.

\section{RESULTADOS}

A amostra foi constituída por dois grupos de crianças de 7 a 14 anos, sendo o GE o grupo com transtorno de processamento auditivo central, com média de idade de 10,1anos $(+-1,7)$ e mediana de 11 anos e o GC, o grupo com avaliação de processamento auditivo normal, com média de idade de 9,9 anos $(+-1,7)$ e mediana de 10 anos $(p$ valor $=0,673)$.

A análise estatística mostrou haver diferença significativa entre os grupos em quase todos os testes de PAC, com exceção do teste de localização sonora (TLS) e teste RGDT. Igualmente, o número de testes de PAC classificados como alterados evidenciaram diferenças estatísticas entre os grupos (p-valor $<0,001)$. O GE teve média de 2,93 testes alterados;

Tabela 1. Valores descritivos para os testes de Processamento Auditivo Central e para o número de testes classificados como alterados

\begin{tabular}{|c|c|c|c|c|c|c|c|c|c|}
\hline Testes PAC & Grupos & Média & Mediana & $\begin{array}{l}\text { Desvio } \\
\text { Padrão }\end{array}$ & Q1 & Q3 & $\mathbf{N}$ & IC & Valor de $p$ \\
\hline \multirow[t]{2}{*}{ TLS } & GE & $97,3 \%$ & $100 \%$ & $7,0 \%$ & $100,0 \%$ & $100,0 \%$ & 15 & $3,6 \%$ & 1,000 \\
\hline & $\mathrm{GC}$ & $97,3 \%$ & $100 \%$ & $7,0 \%$ & $100,0 \%$ & $100,0 \%$ & 15 & $3,6 \%$ & \\
\hline \multirow[t]{2}{*}{ TMSV 4} & GE & $71,1 \%$ & $67 \%$ & $30,5 \%$ & $66,7 \%$ & $100,0 \%$ & 15 & $15,4 \%$ & $0,007^{*}$ \\
\hline & $\mathrm{GC}$ & $95,6 \%$ & $100 \%$ & $11,7 \%$ & $100,0 \%$ & $100,0 \%$ & 15 & $5,9 \%$ & \\
\hline \multirow[t]{2}{*}{ TMSnV 4} & GE & $55,6 \%$ & $67 \%$ & $24,1 \%$ & $33,3 \%$ & $66,7 \%$ & 15 & $12,2 \%$ & $<0,001^{*}$ \\
\hline & $\mathrm{GC}$ & $95,6 \%$ & $100 \%$ & $11,7 \%$ & $100,0 \%$ & $100,0 \%$ & 15 & $5,9 \%$ & \\
\hline \multirow[t]{2}{*}{ TPD } & GE & $38,7 \%$ & $30 \%$ & $18,5 \%$ & $30,0 \%$ & $55,0 \%$ & 15 & $9,3 \%$ & $<0,001^{*}$ \\
\hline & $\mathrm{GC}$ & $93,3 \%$ & $90 \%$ & $6,2 \%$ & $90,0 \%$ & $100,0 \%$ & 15 & $3,1 \%$ & \\
\hline \multirow[t]{2}{*}{ RGDT } & GE & 16,6 & 10,5 & 20,3 & 5,5 & 20 & 15 & 10,3 & 0,105 \\
\hline & $\mathrm{GC}$ & 7,2 & 7,5 & 2,7 & 4,5 & 10 & 15 & 1,4 & \\
\hline \multirow[t]{2}{*}{ F/R OD } & GE & $78,5 \%$ & $76 \%$ & $10,8 \%$ & $72,0 \%$ & $86,0 \%$ & 15 & $5,4 \%$ & $0,003^{*}$ \\
\hline & $\mathrm{GC}$ & $90,1 \%$ & $88 \%$ & $6,7 \%$ & $88,0 \%$ & $96,0 \%$ & 15 & $3,4 \%$ & \\
\hline \multirow[t]{2}{*}{ F/R OE } & GE & $80,0 \%$ & $80 \%$ & $11,8 \%$ & $74,0 \%$ & $86,0 \%$ & 15 & $6,0 \%$ & $0,009^{*}$ \\
\hline & $\mathrm{GC}$ & $89,1 \%$ & $88 \%$ & $6,1 \%$ & $86,0 \%$ & $94,0 \%$ & 15 & $3,1 \%$ & \\
\hline \multirow[t]{2}{*}{ TDD OD } & GE & $86,4 \%$ & $88 \%$ & $11,2 \%$ & $75,6 \%$ & $96,3 \%$ & 15 & $5,7 \%$ & $0,001^{*}$ \\
\hline & $\mathrm{GC}$ & $97,8 \%$ & $100 \%$ & $3,8 \%$ & $96,3 \%$ & $100,0 \%$ & 15 & $1,9 \%$ & \\
\hline \multirow[t]{2}{*}{ TDD OE } & GE & $82,1 \%$ & $80 \%$ & $13,0 \%$ & $72,5 \%$ & $92,5 \%$ & 15 & $6,6 \%$ & $<0,001^{*}$ \\
\hline & $\mathrm{GC}$ & $97,8 \%$ & $100 \%$ & $4,0 \%$ & $97,5 \%$ & $100,0 \%$ & 15 & $2,0 \%$ & \\
\hline Número & GE & 2,93 & 3,00 & 1,16 & 2,00 & 4,00 & 15 & 0,59 & $<0,001^{*}$ \\
\hline $\begin{array}{c}\text { de testes } \\
\text { de PAC } \\
\text { ALTERADOS }\end{array}$ & $\mathrm{GC}$ & 0,00 & 0,00 & 0,00 & 0,00 & 0,00 & 15 & $-x-$ & \\
\hline
\end{tabular}

Teste estatístico de Mann-Whitney; - x - = não foi possível utilizar a estatística; *Diferenças estatísticas significantes

Legenda: PAC = processamento auditivo central; GE = grupo estudo; GC = grupo controle; TLS = teste de localização sonora; TMSV = teste de memória para sons verbais; TMSnV = teste de memória para sons não verbais; TPD = teste de padrão de duração; RGDT = Random Gap Detection Test (teste de detecção de intervalos aleatórios); $\mathrm{F} / \mathrm{R} \mathrm{OD}=$ teste de fala com ruído branco à orelha direita; $\mathrm{F} / \mathrm{R} \mathrm{OE}=$ teste de fala com ruído branco à orelha esquerda; TDD OD = teste dicótico de dígitos orelha direita; TDD OE = teste dicótico de dígitos orelha esquerda; Q1 = 1ำ quartil (25\%); Q3 = 3ำ quartil $(75 \%)$; N = número de crianças testadas; IC = intervalo de confiança 
o GC não apresentou nenhum teste alterado, confirmando a representatividade de cada grupo (Tabela 1).

Em relação à ocorrência do EIVE nos grupos GC e GE, o Teste Qui-Quadrado mostrou que 100\% das crianças do GC apresentaram presença do EIVE, em ambas as orelhas. Entretanto, no GE esse fenômeno foi evidenciado em 86,7\%, com 13,3\% de ausência, evidenciando diferença estatística entre os grupos $(\mathrm{p}$-valor $=0,038)$ (Figura 1).

Os resultados do EIVE às orelhas direita $(\mathrm{OD})$ e esquerda (OE), nos grupos com e sem alteração de PAC, evidenciaram diferença significativa entre os grupos na OE, com valores médios de inibição das EOAT menores no grupo estudo, em relação ao grupo controle (Tabela 2).

$\mathrm{Na}$ análise da curva ROC para o EIVE sobre as emissões otoacústicas, verificou-se que a área da curva na $\mathrm{OE}$ foi de 0,773 , com diferença estatística $(p=0,011)$, não ocorrendo o mesmo para a OD; ao ser considerada a curva ROC de ambas as orelhas, a área da curva foi de 0,720 , com significância estatística $(p=0,003)$ (Tabela 3$)$.

As curvas ROC das orelhas direita e esquerda são apresentadas nas Figuras 2 e 3, respectivamente. Em relação ao EIVE sobre as emissões otoacústicas da $\mathrm{OE}$, a curva ROC mostrou que o ponto de corte foi $0,6 \mathrm{~dB}$, com sensibilidade e especificidade de $73 \%$.

$\mathrm{Na}$ análise conjunta das orelhas, a curva ROC evidenciou ponto de corte de $0,55 \mathrm{~dB}$. A análise da curva ROC da orelha direita foi prejudicada pela presença de mais de um ponto de corte (Tabela 4).

\section{DISCUSSÃO}

A distribuição das 30 crianças nos dois grupos foi realizada após a avaliação do PAC, que identificou como grupo estudo as que apresentaram alteração na maioria dos testes comportamentais (Tabela 1). Esse dado foi importante, pois revelou que os grupos eram definitivamente diferentes quanto à maneira como o sistema auditivo processa a informação acústica. Na maioria dos estudos consultados, a amostra de crianças típicas é selecionada em função apenas da ausência de queixas de $\operatorname{PAC}(7,8,10,16,17)$, o que poderia levar a viés amostral. A realização da avaliação do PAC em todas as crianças pode ser considerada como um diferencial importante na condução do presente estudo, separando os indivíduos com e sem alteração de forma mais criteriosa. De fato, as crianças do GE tiveram média de 2,93 testes e mediana de três testes alterados, sendo que $25 \%$ dos casos apresentam quatro ou mais testes alterados, diferenciando-se do GC de forma significativa. Na literatura, inclusive, a severidade do TPAC tem sido diretamente proporcional ao número de testes $\operatorname{alterados}^{(3,18,19)}$.
Os grupos foram pareados em relação à faixa etária, a fim de se obter maior homogeneidade amostral. Vale ressaltar que, no grupo estudo, apenas uma criança pertencia ao sexo feminino, o que já era esperado, pois a literatura relata maior incidência de TPAC no sexo masculino, numa proporção de

\section{Relação do Grupo com EIVE}

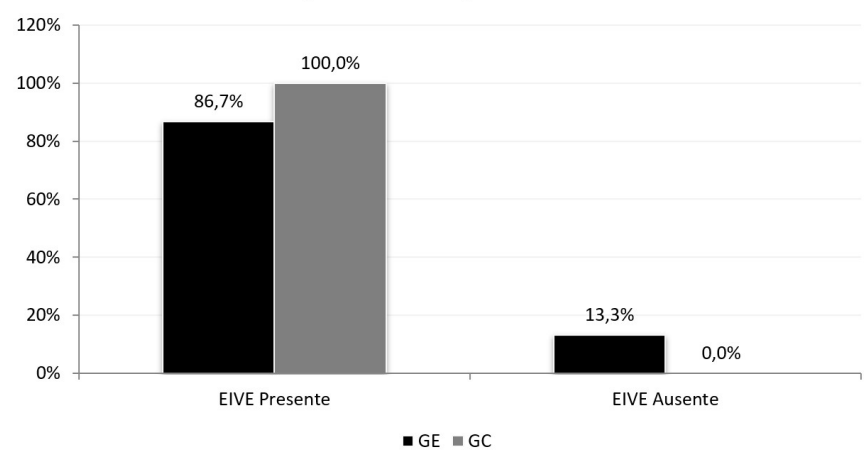

Figura 1. Ocorrência do efeito inibitório da via eferente nos grupos controle e estudo. Teste Qui-Quadrado; $p$ valor 0,038; Diferenças estatísticas significantes

Legenda: EIVE = efeito inibitório da via eferente; $\mathrm{GE}$ = grupo estudo; $\mathrm{GC}$ = grupo controle

\section{Curva ROC}

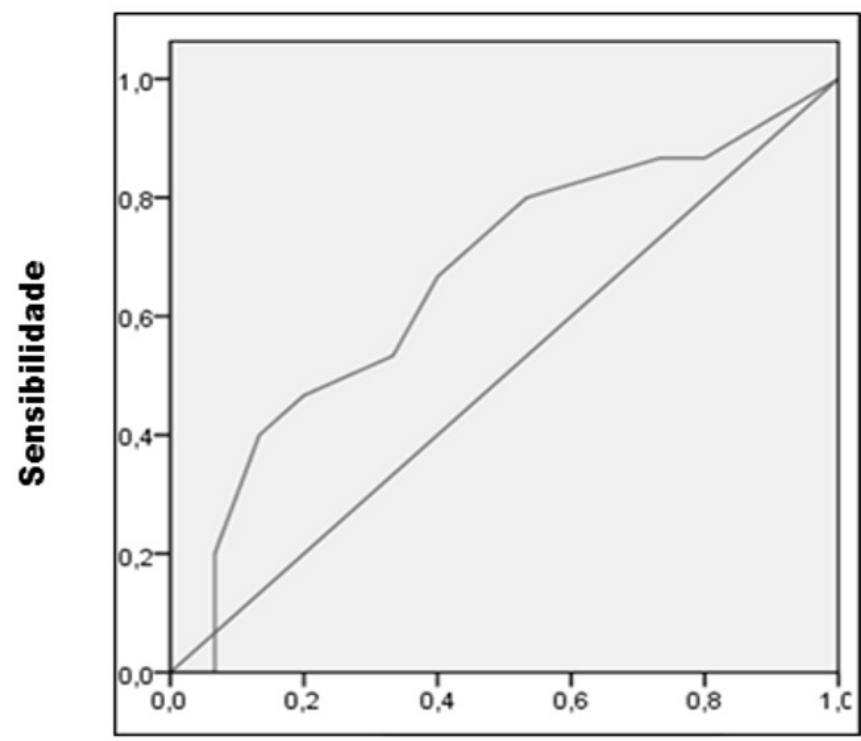

Especificidade

Figura 2. Curva ROC (receiver operator characteristic) do efeito inibitório da via eferente da orelha direita

Tabela 2. Valores descritivos para efeito inibitório da via eferente à orelha direita e à orelha esquerda, nos grupos estudo e controle

\begin{tabular}{ccccccccccc}
\hline \multicolumn{2}{c}{ Grupo } & & Média & Mediana & $\begin{array}{c}\text { Desvio } \\
\text { Padrão }\end{array}$ & Q1 & Q3 & N & IC & Valor de p \\
\hline EIVE OD & GE & 0,55 & 0,50 & 0,32 & 0,35 & 0,70 & 15 & 0,16 & 0,122 \\
& GC & 0,71 & 0,70 & 0,32 & 0,50 & 0,90 & 15 & 0,16 & \\
EIVE OE & GE & 0,41 & 0,30 & 0,38 & 0,15 & 0,60 & 15 & 0,19 & $0,010^{*}$ \\
& GC & 0,87 & 0,80 & 0,48 & 0,50 & 1,20 & 15 & 0,24 & \\
\hline
\end{tabular}

Teste estatístico de Mann-Whitney; *Diferenças estatísticas significantes

Legenda: $\mathrm{GE}$ = grupo estudo; GC = grupo controle; EIVE OD = efeito inibitório da via eferente à orelha direita; EIVE OE = efeito inibitório da via eferente à orelha esquerda; Q1 = 1ำ quartil (25\%); Q3 = 3ำ quartil (75\%); N = número de crianças testadas; IC = intervalo de confiança 


\section{Curva ROC}

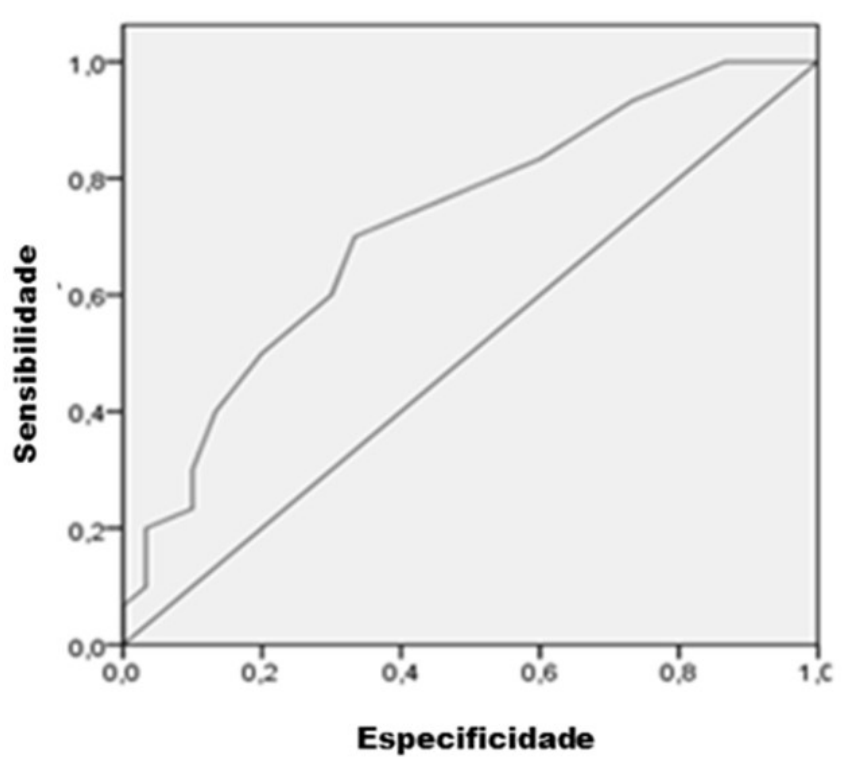

Segmentos diagonais são produzidos por pontos interligados

Figura 3. Curva ROC (receiver operator characteristic) do efeito inibitório da via auditiva eferente da orelha esquerda

Tabela 3. Área da curva ROC (receiver operator characteristic)

\begin{tabular}{ccccc}
\hline EIVE & Área & $\begin{array}{c}\text { Valor } \\
\text { de } \mathbf{p}\end{array}$ & $\begin{array}{c}\text { Limite } \\
\text { inferior }\end{array}$ & $\begin{array}{c}\text { Limite } \\
\text { superior }\end{array}$ \\
\hline OD & 0,664 & 0,125 & 0,465 & 0,864 \\
OE & 0,773 & $\mathbf{0 , 0 1 1}^{*}$ & 0,607 & 0,940 \\
OD + OE & 0,720 & $\mathbf{0 , 0 0 3}^{*}$ & 0,591 & 0,849 \\
\hline
\end{tabular}

${ }^{*}$ Diferença estatística significante

Legenda: $E I V E=$ efeito inibitório da via eferente; $\mathrm{OD}=$ orelha direita; $\mathrm{OE}=$ orelha esquerda
$2: 1^{(3,19)}$. Uma explicação para a maior ocorrência de alteração no sexo masculino seria o fato de a porção posterior do corpo caloso, responsável pela transmissão auditiva e visual entre os hemisférios, ser, geralmente, mais larga e bulbosa em pessoas do sexo feminino e capaz de integrar as informações auditivas e visuais dos dois hemisférios mais efetivamente que em pessoas do sexo masculino ${ }^{(20)}$. Tais diferenças, entretanto, não foram identificadas em outros estudos ${ }^{(21,22)}$.

A prevalência estimada de alteração de PAC na infância, descrita na literatura como em torno de $2 \%$ a $10 \%{ }^{(3)}$, tem impulsionado estudiosos a investigarem o funcionamento da via eferente olivococlear em sujeitos com TPAC, já que a integridade desse sistema permite a adequada localização da fonte sonora, a melhora da escuta no ruído, da sensibilidade e atenção auditivas, dentre outras habilidades ${ }^{(7,17,18,21,22,23,24)}$.

Nesta pesquisa, todos os sujeitos foram submetidos à avaliação auditiva, avaliação do PAC e do EIVE. Todos apresentaram presença de EOAT com clique não linear, caracterizando função coclear normal. Todos foram submetidos à pesquisa do EIVE com estímulos clique lineares, apresentados em blocos de dez segundos, com e sem ruído. Na literatura, estudos recomendam o uso do clique linear como estimulo ativador do EIVE nas $\operatorname{EOAT}^{(7,10,18)}$ por apresentar uma diferença de resposta mais robusta $^{(22)}$. Tal como no presente estudo, diversas pesquisas utilizaram o ruído branco como estímulo supressor ${ }^{(7,10,21,24,25)}$, obtendo melhor comparação entre os achados.

O GE apresentou menor ocorrência do EIVE, em relação ao GC (Figura 1), resultado semelhante aos obtidos na literatura, que evidenciaram menor ocorrência de supressão em crianças com TPAC ${ }^{(6)}$. Além disso, estudos revelaram que a menor ocorrência do EIVE poderia contribuir para maior dificuldade de compreender a fala no ruído e, desta forma, interferir no processo de aprendizagem ${ }^{(7,19,21)}$. De fato, estudo em crianças de 8 a 12 anos com alteração de leitura identificou correlação negativa entre o resultado do EIVE e a relação sinal/ruído

Tabela 4. Coordenadas da curva ROC (receiver operator characteristic), medidas de sensibilidade e especificidade em cada ponto para o efeito inibitório da via auditiva eferente, na orelha esquerda, orelha direita e em ambas as orelhas

\begin{tabular}{|c|c|c|c|c|c|c|c|c|}
\hline $\begin{array}{c}\text { EIVE } \\
\text { OD }\end{array}$ & Sensibilidade & Especificidade & EIVE OE & Sensibilidade & Especificidade & EIVE AO & Sensibilidade & Especificidade \\
\hline$-0,8$ & $100,0 \%$ & $0,0 \%$ & $-1,2$ & $100,0 \%$ & $0,0 \%$ & $-1,20$ & $100,0 \%$ & $0,0 \%$ \\
\hline 0,3 & $86,7 \%$ & $20,0 \%$ & $-0,1$ & $100,0 \%$ & $6,7 \%$ & $-0,10$ & $100,0 \%$ & $3,3 \%$ \\
\hline 0,4 & $86,7 \%$ & $26,7 \%$ & 0,1 & $100,0 \%$ & $13,3 \%$ & 0,05 & $100,0 \%$ & $6,7 \%$ \\
\hline 0,5 & $80,0 \%$ & $46,7 \%$ & 0,2 & $100,0 \%$ & $26,7 \%$ & 0,15 & $100,0 \%$ & $13,3 \%$ \\
\hline 0,6 & $66,7 \%$ & $60,0 \%$ & 0,3 & $100,0 \%$ & $33,3 \%$ & 0,25 & $93,3 \%$ & $26,7 \%$ \\
\hline 0,7 & $53,3 \%$ & $66,7 \%$ & 0,4 & $80,0 \%$ & $53,3 \%$ & 0,35 & $83,3 \%$ & $40,0 \%$ \\
\hline 0,8 & $46,7 \%$ & $80,0 \%$ & 0,5 & $73,3 \%$ & $60,0 \%$ & 0,45 & $76,7 \%$ & $53,3 \%$ \\
\hline 0,9 & $40,0 \%$ & $86,7 \%$ & 0,6 & $73,3 \%$ & $73,3 \%$ & 0,55 & $70,0 \%$ & $66,7 \%$ \\
\hline 1,0 & $20,0 \%$ & $93,3 \%$ & 0,7 & $66,7 \%$ & $73,3 \%$ & 0,65 & $60,0 \%$ & $70,0 \%$ \\
\hline 1,1 & $13,3 \%$ & $93,3 \%$ & 0,8 & $53,3 \%$ & $80,0 \%$ & 0,75 & $50,0 \%$ & $80,0 \%$ \\
\hline 1,3 & $0,0 \%$ & $93,3 \%$ & 0,9 & $40,0 \%$ & $86,7 \%$ & 0,85 & $40,0 \%$ & $86,7 \%$ \\
\hline \multirow[t]{8}{*}{2,4} & $0,0 \%$ & $100,0 \%$ & 1,1 & $33,3 \%$ & $86,7 \%$ & 0,95 & $30,0 \%$ & $90,0 \%$ \\
\hline & & & 1,2 & $26,7 \%$ & $100,0 \%$ & 1,05 & $23,3 \%$ & $90,0 \%$ \\
\hline & & & 1,4 & $20,0 \%$ & $100,0 \%$ & 1,15 & $20,0 \%$ & $96,7 \%$ \\
\hline & & & 1,5 & $13,3 \%$ & $100,0 \%$ & 1,25 & $13,3 \%$ & $96,7 \%$ \\
\hline & & & 1,7 & $6,7 \%$ & $100,0 \%$ & 1,35 & $10,0 \%$ & $96,7 \%$ \\
\hline & & & 2,8 & $0,0 \%$ & $100,0 \%$ & 1,50 & $6,7 \%$ & $100,0 \%$ \\
\hline & & & & & & 1,70 & $3,3 \%$ & $100,0 \%$ \\
\hline & & & & & & 2,80 & $0,0 \%$ & $100,0 \%$ \\
\hline
\end{tabular}

Legenda: EIVE OD = efeito inibitório da via eferente à orelha direita; EIVE OE = efeito inibitório da via eferente à orelha esquerda; EIVE AO = efeito inibitório da via eferente em ambas as orelhas 
necessária para o reconhecimento de palavras ${ }^{(7)}$. As crianças com EIVE elevado reconheciam palavras com relação sinal/ruído $\operatorname{menor}^{(7)}$.

Por outro lado, estudo recente ${ }^{(16)}$ não identificou diferenças entre crianças com dificuldades de escuta e TPAC e crianças com desenvolvimento típico. Os autores atribuíram as diferenças entre seus resultados e os de outros estudos como decorrentes de diferenças metodológicas na avaliação do EIVE, da heterogeneidade nos deficit de PAC que fundamentam o TPAC e da falta de medidas rigorosas no tratamento dos dados ${ }^{(16,24)}$.

Ao comparar os valores médios do EIVE entre os grupos, constatou-se que o GE apresentou menor redução da amplitude de resposta com ruído contralateral, com diferença significativa à orelha esquerda (Tabela 2). Esses achados confirmam os encontrados na literatura, indicando que as crianças com TPAC apresentam diminuição da função inibitória eferente por alteração do sistema olivococlear medial, o que pode afetar a habilidade de escuta no ruído ${ }^{(6,8,10)}$.

No presente estudo, os valores de EIVE encontrados no GC variaram de $0,2 \mathrm{~dB}$ a 1,8 $\mathrm{dB}$ com valores médios de $0,71 \mathrm{~dB}$ na orelha direita e $0,87 \mathrm{~dB}$ na orelha esquerda (Tabela 2), similarmente aos obtidos em normo-ouvintes (entre $0,6 \mathrm{~dB}$ e $1,62 \mathrm{~dB})^{(6,7,26)}$. No GE, os valores de EIVE variaram de $0,1 \mathrm{~dB}$ a $1,4 \mathrm{~dB}$, com valores médios de $0,55 \mathrm{~dB}$ na orelha direita $\mathrm{e}$ de $0,41 \mathrm{~dB}$ na orelha esquerda, inferiores aos obtidos no grupo controle, com diferença estatisticamente significativa na orelha esquerda (Tabela 2).

Os valores obtidos nas crianças com transtorno de processamento auditivo no presente estudo foram inferiores aos descritos na literatura, em crianças com dificuldades de leitura $^{(7)}$ e em crianças com dificuldades de aprendizagem ${ }^{(26,27)}$.

Os valores de EIVE foram mais elevados na orelha direita no grupo estudo, assim como encontrado em estudo de crianças com TPAC, revelando assimetria do sistema eferente com melhor sensibilidade da orelha direita ${ }^{(9)}$ e já descrita na literatura ${ }^{(9)}$. A resposta de supressão assimétrica entre as duas orelhas poderia sugerir que essa assimetria em indivíduos normais representaria a lateralização na função das células ciliadas externas, indicando que essas células podem ser mais eficientes ou reativas nessa orelha ${ }^{(7,9)}$, que se destaca como dominante, pois leva as informações auditivas para o hemisfério esquerdo que processa a linguagem ${ }^{(7,9)}$. Da mesma forma, o sistema eferente protege mais a orelha direita, que é dominante.

Os resultados do presente estudo apontaram que o efeito inibitório diferiu entre os grupos estudados somente na orelha esquerda. Esse dado, de maneira indireta, confirma a assimetria auditiva periférica com melhores respostas à direita, no grupo estudo. Alguns autores apontaram a diferença nos padrões do EIVE entre a OD e OE, descrevendo assimetria interaural e ressaltando melhor sensibilidade da OD, aliada a menor suscetibilidade a lesões por ruído ${ }^{(9,18)}$.

No presente estudo, os resultados obtidos com a curva ROC (Tabela 3), técnica utilizada para estabelecer um ponto de corte para medidas de sensibilidade e especificidade do teste diagnóstico, revelaram que a área da curva ROC foi de 0,664 na OD, de 0,773 na OE e de 0,720, considerando-se ambas as orelhas. Conclui-se, portanto, que houve resultado estatisticamente significativo somente na curva ROC para a supressão na orelha esquerda $(\mathrm{OE})$ e para ambas as orelhas, pois o valor abaixo da curva foi estatisticamente diferente de 0,5 .

De acordo com Hanley \& $\mathrm{McNeil}^{(15)}$, uma curva ROC com área entre 0.7 e 0.9 está associada com um teste considerado "bom". Sendo assim, tendo em vista a área da curva ROC que foi encontrada no presente estudo, em relação ao EIVE, pode-se afirmar que o EIVE constitui um índice próximo ao que se considera bom, em termos de sensibilidade e especificidade.

Se considerarmos apenas os resultados da orelha esquerda, os dados obtidos na curva ROC em relação ao EIVE permitiram identificar o ponto de corte 0,6 . Ou seja, valores de efeito inibitório da via eferente acima de 0,6 indicaram, com 73,3\% de sensibilidade e especificidade, a possibilidade desse indivíduo apresentar processamento auditivo normal (Tabela 4). Na análise conjunta das orelhas, a curva ROC evidenciou que o ponto que potencializa a sensibilidade e especificidade é 0,55 . Ou seja, valores de EIVE acima de 0,55 diagnosticaram uma pessoa com a possibilidade de apresentar avaliação de processamento auditivo normal, com $70,0 \%$ de sensibilidade e $66,7 \%$ de especificidade. Portanto, ao considerar ambas as orelhas, observam-se valores muito próximos àqueles obtidos com a realização da curva ROC somente na orelha esquerda (Tabela 4).

Dessa forma, crianças com valores de EIVE inferiores a 0,55 podem apresentar maior risco de alteração de PAC e, quanto menor esse valor, maior a probabilidade da alteração, dado que valoriza a inclusão da investigação do EIVE na bateria de testes de processamento auditivo, conforme recomendado ${ }^{(6,27)}$. As medidas de sensibilidade e especificidade são descritas como instrumento precioso para uma avaliação diagnóstica. Tais informações são fundamentais à precisão do diagnóstico, pois níveis de sensibilidade e/ou especificidade muito abaixo do esperado para determinada ferramenta diagnóstica válida podem levar a conclusões errôneas e, possivelmente, a intervenções inadequadas. Em geral, são esperados valores de sensibilidade e especificidade próximos de $80 \%$, para que os resultados sejam considerados uma medida válida do desempenho da criança ${ }^{(28)}$. Uma limitação deste estudo foi que não se atingiu sensibilidade e especificidade acima de $80 \%$, fato que poderia estar relacionado ao tamanho reduzido da amostra. Novos estudos com amostras mais amplas poderiam vir a confirmar os achados.

No presente estudo, pôde-se identificar menor ocorrência do efeito inibidor do sistema eferente no grupo de crianças com transtorno de processamento auditivo, com valores inferiores aos do grupo controle, em concordância com os achados da literatura $^{(6,7,21,27)}$. A dificuldade de processamento dos sinais de fala na presença de ruído competitivo, queixa tão comum em indivíduos com TPAC, poderia também estar relacionada à alteração da função inibitória do sistema eferente.

Tais resultados sugerem que a avaliação do EIVE, procedimento rápido, indolor e seguro, seja incluída nos protocolos de avaliação do processamento auditivo, a fim de reforçar e tornar a avaliação mais precisa e confiável e, consequentemente, promover diagnóstico e intervenção oportunos. A associação entre os resultados da avaliação do PAC e funcionamento do sistema eferente também direcionam para recomendação de inclusão da avaliação do sistema eferente no protocolo de avaliação do PAC.

A discussão e relevância sobre o tema tornam-se evidentes, visto que propostas e protocolos de avaliação de PAC vêm crescendo na literatura ${ }^{(28-30)}$.

\section{CONCLUSÃO}

Houve menor ocorrência do efeito inibitório da via eferente sobre as emissões otoacústicas em crianças com transtorno do processamento auditivo central. O efeito inibitório da via eferente diferiu entre as orelhas, com pior desempenho à esquerda. 
O valor recomendado para o ponto de corte em relação ao efeito inibitório da via auditiva eferente é de $0,55 \mathrm{~dB}$. Quanto mais elevado esse valor, menor chance de a criança apresentar transtorno de processamento auditivo central. Um efeito inibitório da via auditiva eferente inferior a $0,55 \mathrm{~dB}$ indica maior probabilidade de ocorrência desse transtorno.

\section{REFERÊNCIAS}

1. Azevedo MF, Angrisani RG. Desenvolvimento das habilidades auditivas. In: Boéchat EM, Menezes PL, Couto CM, Frizzo ACF, Sharlach RC, Anastasio ART, organizadores. Tratado de Audiologia. 2a ed. Rio de Janeiro: Guanabara Koogan; 2015. p. 373-80.

2. Guinan JJ Jr. Cochlear mechanics, otoacoustic emissions, and medial olivocochlearefferents: twenty years of advances and controversies along with areas ripe for New York. In: Popper AN, Fay RR, editors. Perspectives on auditory research. New York: Springer; 2014. p. 22946. http://dx.doi.org/10.1007/978-1-4614-9102-6_13.

3. ASHA: American Speech-Language-Hearing Association [Internet]. Rockiville: American Speech-Language-Hearing Association; c20052017 [citado em 2020 Nov 13]. Disponível em: www.asha.org/policy/ TR2005-00043/.html.

4. Guinan JJ Jr, Backus BC, Lilaonitkul W, Aharonson V. Medial olivocochlear efferent reflex in humans: otoacoustic emission (OAE) measurement issues and the advantages of stimulus frequency OAES. J Assoc Res Otolaryngol. 2003;4(4):521-40. http://dx.doi.org/10.1007/ s10162-002-3037-3. PMid:12799992.

5. Arcuri CF, Schiefer AM, Azevedo MF. Pesquisa do efeito de supressão e do processamento auditivo em indivíduos que gaguejam. CoDAS. 2017;29(3):e20160230. http://dx.doi.org/10.1590/2317-1782/20172016230. PMid:28538833.

6. Muchnik C, Ari-Even Roth DAE, Othman-Jebara R, Putter-Katz H, Shabtai EL, Hildesheimer M. Reduced medial olivocochlear bundle system function in children with auditory processing disorders. Audiol Neurootol. 2004;9(2):107-14. http://dx.doi.org/10.1159/000076001. PMid: 14981358 .

7. Akbari M, Panahi R, Valadbeigi A, Nahrani MH. Speech-in-noise perception ability can be related to auditory efferent pathway function: a comparative study in reading impaired and normal reading children. Braz J Otorhinolaryngol. 2020;86(2):209-16. http://dx.doi.org/10.1016/j. bjorl.2018.11.010. PMid:30772249.

8. Burguetti FAR, Carvallo RMM. Sistema auditivo eferente: efeito no processamento auditivo. Braz J Otorhinolaryngol. 2008;74(5):737-45. https://doi.org/10.1590/S0034-72992008000500016.

9. Bidelman GM, Bhagat SP. Right-ear advantage drives the link between olivocochlear efferent 'antimasking' and speech-in-noise listening benefits. Neuroreport. 2015;26(8):483-7. http://dx.doi.org/10.1097/ WNR.0000000000000376. PMid:25919996.

10. Boothalingam S, Allan C, Allen P, Purcell D. Cochlear delay and medial olivocochlear functioning in children with suspected auditory processing disorder. PLoS One. 2015;10(8):e0136906. http://dx.doi. org/10.1371/journal.pone.0136906. PMid:26317850.

11. Jerger J. Clinical experience with impendance audiometry. Arch Otolaryng. 1970;92(4):311-24.

12. Pereira LD. Processamento auditivo central: abordagem passo a passo. In: Pereira LD, Schochat E, organizadores. Processamento auditivo central: manual de avaliação. São Paulo: Lovise; 1997. p. 49-59.

13. Keith RW. Random gap detection test. Saint Louis: Auditec; 2000.
14. Collet L, Veuillet E, Bene J, Morgan A. Effects of contralateral white noise on click-evoked emissions in normal and sensorineural ears: towards an exploration of the medial olivocochlear system. Audiology. 1992;31(1):1-7. http://dx.doi.org/10.3109/00206099209072897. PMid:1554329.

15. McNeil BJ, Hanley JA. Statistical approaches to the analysis of receiver operating characteristic (ROC) curves. Med Decis Making. 1984;4(2):137-50. http://dx.doi.org/10.1177/0272989X8400400203. PMid:6472062.

16. Boothalingam S, Allan C, Allen P, Purcell DW. The medial olivocochlear reflex is unlikely to play a role in listening difficulties in children. Trends Hear. 2019;23:2331216519870942. http://dx.doi. org/10.1177/2331216519870942. PMid:31558110.

17. Tokgoz-Yilmaz S, Kose SK, Turkyilmaz MD, Atay G. The role of the medial olivocochlear system in the complaints of understanding speech in noisy environments by individuals with normal hearing. Auris Nasus Larynx. 2013;40(6):521-4. http://dx.doi.org/10.1016/j. anl.2013.04.003. PMid:23694738.

18. Iliadou VV, Weihing J, Chermak GD, Bamiou DE. Otoacoustic emission suppression in children diagnosed with central auditory processing disorder and speech in noise perception deficits. Int $\mathrm{J}$ Pediatr Otorhinolaryngol. 2018;111:39-46. http://dx.doi.org/10.1016/j. ijporl.2018.05.027. PMid:29958612.

19. Mishra SK. Medial efferent mechanisms in children with auditory processing. Front Hum Neurosci. 2014;8:860. http://dx.doi.org/10.3389/ fnhum.2014.00860. PMid:25386132.

20. Guinan JJ Jr. Olivocochlearefferents: anatomy, physiology, function, and the measurement of efferent effects in humans. Ear Hear. 2006;27(6):589-607. http://dx.doi.org/10.1097/01.aud.0000240507.83072. e7. PMid: 17086072.

21. Jedrzejczak WW, Pilka E, Skarzynski PH, Skarzynski H. Contralateral suppression of otoacoustic emissions in pre-school children. Int J Pediatr Otorhinolaryngol. 2020;132:109915. http://dx.doi.org/10.1016/j. ijporl.2020.109915. PMid:32028191.

22. Mattsson TS, Lind O, Follestad T, Grøndahl K, Wilson W, Nordgård S. Contralateral suppression of otoacoustic emissions in a clinical sample of children with auditory processing disorder. Int J Audiol. 2019;58(5):301-10. http://dx.doi.org/10.1080/14992027.2019.15703 58. PMid:30849269.

23. Kalaiah MK, Theruvan NB, Kumar K, Bhat JS. Role of active listening and listening effort on contralateral suppression of transient evoked otoacousic emissions. J Audiol Otol. 2017;21(1):1-8. http://dx.doi. org/10.7874/jao.2017.21.1.1. PMid:28417101.

24. Mertes IB, Wilbanks EC, Leek MR. Olivocochlear efferent activity is associated with the slope of the psychometricfunction of speech recognition in noise. Ear Hear. 2018;39(3):583-93. http://dx.doi. org/10.1097/AUD.0000000000000514. PMid:29135685.

25. Kalaiah MK, Nanchirakal JFL, Kharmawphlang L, Noronah SC. Contralateral suppression of transient evoked otoacoustic emissions for various noise signals. Hear Balance Commun. 2017;15(2):84-90. http://dx.doi.org/10.1080/21695717.2017.1311504.

26. Aksoy ED, Culhaoğlu B, Öcal FCA, Erbek SS, Erbek HS. Does the efferent auditory system have a role in children with specific learning disabilities? Turk Arch Otorhinolaryngol. 2019;57(1):30-3. http:// dx.doi.org/10.5152/tao.2019.3748. PMid:31049250.

27. Rocha-Muniz CN, Mamede Carvallo RM, Schochat E. Medial olivocochlear function in children with poor speech-in-noise performance and language disorder. Int J Pediatr Otorhinolaryngol. 2017;96:116-21. http://dx.doi.org/10.1016/j.ijporl.2017.03.003. PMid:28390599. 
28. Jerger J, Musiek F. Report of the consensus conference on the diagnosis of auditory processing disorders in school-aged children. J Am Acad Audiol. 2000;11(9):467-74. PMid:11057730.

29. Skarzynski PH, Wlodarczyk AW, Kochanek K, Pilka A, Jedrzejczak WW, Olszewski L, et al. Central auditory processing disorder (CAPD): tests in a school-age hearing screening programme - analysis of 76,429 children. Ann Agric Environ Med. 2015;22(1):90-5. http://dx.doi. org/10.5604/12321966.1141375. PMid:25780835.

30. Carvalho NG, Ubiali T, Amaral MIR, Colella-Santos MF. Procedures for central auditory processing screening inschoolchildren. Braz J Otorhinolaryngol. 2019;85(3):319-28. http://dx.doi.org/10.1016/j. bjorl.2018.02.004. PMid:29615299. 